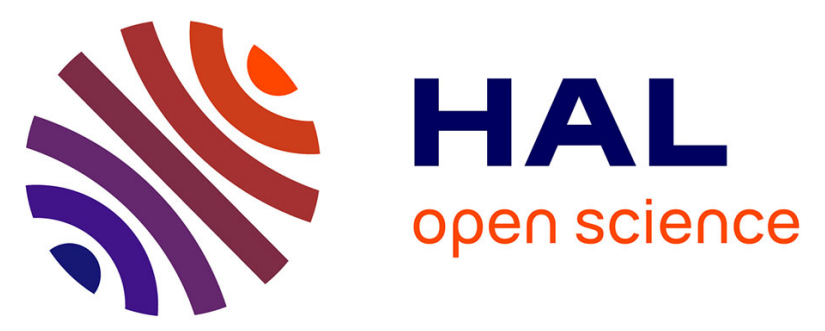

\title{
Vorinostat and Mithramycin A in combination therapy as an interesting strategy for the treatment of Sézary $T$ lymphoma: a transcriptomic approach
}

R. Ragheb, G. Venton, R. Chelbi, N. Bonnet, T. Le Treut, V. Ivanov, C. Mercier, P. Poulin, N. Beaufils, J. Gabert, et al.

\section{To cite this version:}

R. Ragheb, G. Venton, R. Chelbi, N. Bonnet, T. Le Treut, et al.. Vorinostat and Mithramycin A in combination therapy as an interesting strategy for the treatment of Sézary T lymphoma: a transcriptomic approach. Archives of Dermatological Research, 2017, 309 (8), pp.611-623. 10.1007/s00403017-1761-0 . hal-01595894

\section{HAL Id: hal-01595894 \\ https://hal-amu.archives-ouvertes.fr/hal-01595894}

Submitted on 28 May 2018

HAL is a multi-disciplinary open access archive for the deposit and dissemination of scientific research documents, whether they are published or not. The documents may come from teaching and research institutions in France or abroad, or from public or private research centers.
L'archive ouverte pluridisciplinaire HAL, est destinée au dépôt et à la diffusion de documents scientifiques de niveau recherche, publiés ou non, émanant des établissements d'enseignement et de recherche français ou étrangers, des laboratoires publics ou privés. 


\title{
Vorinostat and Mithramycin $A$ in combination therapy as an interesting strategy for the treatment of Sézary $T$ lymphoma: a transcriptomic approach
}

\author{
R. Ragheb,G. Venton,R. Chelbi,N. Bonnet,T. Le Treut,V. Ivanov,C. Mercier,P. Poulin \\ N. Beaufils,J. Gabert,P. Suchon,P. Rihet,B. Loriod,B. Kahn-Perlès,Régis T. Costello \\ 1.Aix Marseille Université, INSERM UMR_S 1090, Technological Advances for \\ Genomics and ClinicsMarseille-LuminyFrance
}

2.Assistance Publique des Hôpitaux de Marseille, Hôpital de la Conception, Service d'Hématologie et de Thérapie CellulaireMarseilleFrance

3.Assistance Publique Hôpitaux de Marseille, Hôpital Nord, Service de DermatologieMarseilleFrance

4.Assistance Publique Hôpitaux de Marseille, Hôpital Nord, Laboratoire d'HématologieMarseilleFrance

5.Assistance Publique Hôpitaux de Marseille, Hôpital La Conception, Service d'HémaphérèseMarseilleFrance

6.Assistance Publique des Hôpitaux de Marseille, Hôpital Nord, Laboratoire de Biochimie Biologie MoléculaireMarseilleFrance

7.Assistance Publique des Hôpitaux de Marseille, Hôpital de la Timone, Service d'HématologieMarseilleFrance

\begin{abstract}
SAHA (vorinostat) is a histone deacetylase inhibitor approved by the USA Food and Drug Administration (FDA) for treating advanced refractory cutaneous T cell lymphomas. As SAHA alters the expression of many genes under control of the $\mathrm{Sp} 1$ transcription factor, we examined the effect of its association with the FDA-approved anticancer antibiotic Mithramycin A (MTR, plicamycin), a competitive inhibitor of Sp1 binding to DNA. Sézary syndrome (SS) cells, expanded ex vivo from peripheral blood mononuclear cells of 4 patients, were tested for their sensitivity to the drugs regarding cytotoxicity and differential responsive gene expression. Multivariate statistical methods were used to identify genes whose expression is altered by SAHA, MTR, and the synergist effect of the two drugs. MTR, like SAHA, induced the apoptosis of SS cells, while the two drugs in combination showed clear synergy or potentiation. Expression data stressed a likely important role of additive or synergistic epigenetic modifications in the combined effect of the two drugs, while direct inhibition of Sp1-dependent transcription seemed to have only limited impact. Ontological analysis of modified gene expression suggested that the two drugs, either independently or
\end{abstract}


synergistically, counteracted many intertwined pro-survival pathways deregulated in SS cells, resistance of these tumors to intrinsic and extrinsic apoptosis, abnormal adhesion migration, and invasive properties, as well as immunosuppressive behavior. Our findings provide preliminary clues on the individual and combined effects of SAHA and MTR in SS cells and highlight a potential therapeutic interest of this novel pair of drugs for treatment of SS patients.

\section{Keywords}

Sézary syndrome SAHA Mithramycin A Synergistic apoptosis Gene expression profiling

R. Ragheb and G. Venton contributed equally to this work.

\section{Introduction}

Sézary syndrome (SS) is an aggressive epidermotropic cutaneous T cell lymphoma (CTCL) defined by the triad of erythroderma, generalized lymphadenopathy, and the presence of clonally related neoplastic T cells with cerebriform nuclei (Sézary cells) in skin, lymph nodes, and peripheral blood (PB) (more than $1 \mathrm{~g} / \mathrm{L}$ lymphoma cells in $\mathrm{PB}$, an expanded $\mathrm{CD}^{+} \mathrm{T}$ cell population resulting in a CD4/CD8 ratio of more than $10 \mathrm{and} /$ or loss of one or more $\mathrm{T}$ cell antigens) [5]. SS cells show a clonal rearrangement of T cell receptor (TCR), have evolved toward T helper 2 (Th2) phenotype resulting in an increased production of interleukin IL-4, IL-5, and IL-10 [21], have a central memory profile (L-selectin/CD62L ${ }^{+}$) which distinguishes it from that of mycosis fungoïde (MF) CTCL subtype [4], and display a Treg phenotype with a suppressive function in a substantial number of patients [20]. Despite the variety of therapeutic treatments that are presently offered to Sézary patients, none of them is curative and their goal is to maintain a long-term response and preserve life quality. Improved therapy deserves a better knowledge of the pathogenic mechanisms underlying the SS syndrome and of the mechanisms associated to the resistance to treatments. Microarray-based and RNA-Seq gene expression profiling of SS cells have identified and validated candidate genes as novel diagnostic markers and putative therapeutic targets [9].

Histone deacetylase inhibitors (HDACis) are recent anticancer drugs showing efficacy in hematologic and solid cancer. They can induce tumor cell death, cell cycle arrest, and/or differentiation mostly by transcription-dependant mechanisms leading to the accumulation of acetylated histones and non-histone proteins. Suberoylanilide hydroxamic acid (SAHA, Vorinostat, marketed as Zolinza ${ }^{\circledR}$ ) is a pan-HDAC inhibitor which causes growth arrest as well as caspase-dependent apoptotic and caspase-independent autophagic cell death [30] in a great variety of tumor types. An overall response rate of $29.7 \%$ has been reported in refractory CTCL, which led to the approval of this drug by the USA Food and Drug Administration (FDA) for these pathologies [12]. These compounds need to be combined to other anticancer agents to increase their efficacy in CTCLs. A recent gene expression profiling performed following SAHA treatment of several CTCL lines has underlined the potential benefit of combining this agent to inhibitors targeting PI3K [32]. Moreover, a case study on refractory SS reported significant clinical improvement following treatment with SAHA combined to interferon gamma [13].

In our study, we hypothesized that the efficacy of SAHA should be increased by combining it with a drug that acts directly on its transcriptional regulatory properties. As with several other 
HDACis, SAHA modifies the expression of many genes under control of Sp1 (or Sp3) transcription factors via distinct gene- and cellular context-dependent mechanisms. SAHA induces a repression of survival genes [17] and expression of antineoplastic genes [16]. However, reports have indicated that SAHA could also induce the expression of some key survival genes under control of Sp1 [22]. Given that similar modulations could limit the effectiveness of SAHA in SS, we tested here its association with the anticancer antibiotic Mithramycin (Plicamycin, MTR, marketed as Mithracin ${ }^{\circledR}$ ), a direct inhibitor of the binding of Sp1 family factors to GC-rich promoters [3]. We report that MTR, similarly to SAHA, induced the apoptotic death of SS cells. The two drugs clearly demonstrated a synergistic effect that could even overcome the resistance to MTR used in monotherapy. This finding is of major clinical interest since it could allow the inhibition of the development of chemotherapy-resistant clones during treatment, may offer second- or third-line treatment to relapsed patients, and minimize the deleterious dose-dependent effect of each drug used as monotherapy. Moreover, using whole genome expression profiling, we provide some clues on the molecular mechanisms underlying the lethal effect of the two drugs and their synergistic behavior.

\section{Methods}

\section{Cells and culture conditions}

PBMCs were collected from 4 SS patients following informed written consent and approval of the study by the "Comité de Protection des Personnes" (CPP) Sud-Méditerranée II. Patient characteristics and flow cytometry (FC) immunophenotypic data (FACS Navios cytometer/Beckman Coulter, Miami Florida, USA) of the samples are detailed in Supplementary Data, Table S1. FC analysis of TCR-V $\beta$ expression was assessed using a panel of 24 monoclonal antibodies known to react with specific TCR-V $\beta$ families according to the manufacturer's recommendations (IOTest Beta Mark TCR-V $\beta$ Repertoire Kit; Beckman Coulter). The high percentage of T lymphocytes (over $70 \%$ of white blood cell count) and the great majority of $\mathrm{CD}^{+}$among these (around 90\%) led us to put samples in culture without prior purification. SS cells were expanded for 7 days in RPMI medium supplemented with 10\% FCS, in the presence of recombinant IL-7 (10 ng/mL, Bio Basic Inc., Ontario, Canada) and anti-CD28 (monoclonal antibody 248 ascites from Dr. A. Moretta, Genova provided to us by Dr. D. Olive, Marseille) according to culture conditions reported in the literature [10].

\section{Cell treatments and assessment of synergy}

SAHA was purchased from Cayman Chemical (Ann Arbor, MI, USA), MTR from SigmaAldrich (St. Louis, MO, USA), and 7-AAD and annexin V-APC from BD Pharmingen (France). Patient cells were treated with increasing doses of SAHA or MTR and the ED50 of each drug was determined as the dose of treatment inducing $50 \%$ apoptosis after $48 \mathrm{~h}$. Viable and apoptotic cells were identified by flow cytometry (BD FACSCalibur) as annexin $\mathrm{V}^{-} / 7$ $\mathrm{AAD}^{-}$and annexin $\mathrm{V}^{+} / 7-\mathrm{AAD}^{-}{ }^{+}{ }^{+}$, respectively. Combined effects of SAHA plus MTR were determined according to the method of Chou and Talalay [7] that assesses first the apoptotic response of cells to fractionated cascade doses of the ED50 of each drug (alone or in combination) and, second, the combination index (CI) deduced from dose-response curves (CalcuSyn software, Biosoft, Cambridge, GB). The method of Chou and Talalay permits to 
determine the dose reduction index (DRI) that represents the order of magnitude (fold) of dose reduction that is allowed for a given degree of effect compared with the drug alone.

\section{Microarray processing (RNA isolation, amplification, and hybridization)}

RNA extraction was performed with RNeasy ${ }^{\circledR}$ mini Kit (Qiagen GmbH, Hilden, Germany). On-column digestion of DNA was done during RNA purification with RNase-free DNase set (Qiagen GmBH). Quantification of RNA was achieved using a NanoDrop ND-1000 UV-Vis spectrophotometer (Thermo Fisher Scientific, Illkirch, France) and the quality of the RNA samples RNA (Integrity Number over 8) was analyzed with the Bioanalyzer 2100 (Agilent Technologies ${ }^{\circledR}$, massy, France) used with Agilent chips (RNA Nano Chip ${ }^{\circledR}$ ). Samples and microarrays were processed according to Agilent's recommendations. In brief, total RNA was reverse-transcribed with AffinityScript RT enzyme and cDNA was in vitro transcribed and mono-labeled using T7 RNA polymerase and Cy3-CTP, before fragmentation of cRNA and hybridization (600 ng per sample $/ 17 \mathrm{~h} / 65^{\circ} \mathrm{C}$ ) to a SurePrint G3 Human GE $8 \times 60 \mathrm{k}$ microarray. After washing, the microarrays were scanned with a SureScan Agilent Microarray Scanner.

\section{Microarray experimental design and data analysis}

We used a fully crossed factorial design that combined each state of one factor to each state of other factors. This led to 48 samples, corresponding to 4 patients, 4 treatments, and 3 replicates. Quantification of signals was performed with Agilent's Feature Extraction Software. From the raw data, data filtration, quantile normalization, probe averaging, and data backup were performed using the R statistical software LIMMA package [28]. For high confidence, two statistical models were used to establish the sets of genes differentially responsive to drug treatments, i.e., Two-Way ANOVA (geneANOVA) [11] and Multivariate Linear Regression (library LIMMA; R) tests. The factors used were SAHA (two groups, i.e., SAHA samples and NON-SAHA samples) and MTR (two groups, i.e., MTR samples and NON-MTR samples). We also took into account the interaction of those two factors (SAHA:MTR), allowing us to look for genes responding differentially in (SAHA + MTR) samples when compared to control, SAHA, and MTR samples. Also, we explicitly separated the effect of each drug and the one of the interaction between drugs by writing the statistical model for each gene: $Y_{\mathrm{i}}=\alpha+\beta_{1} S+\beta_{2} M+\beta_{3} S M+\varepsilon \mathrm{i}$, where $Y_{\mathrm{i}}$ is the expression level for gene i, $S$ is the SAHA factor, $M$ is the MTR factor, and $\varepsilon_{\mathrm{i}}$ is the residual of the model. Moreover, multiple testing corrections were performed using False Discovery Rates (FDRs) of $0.5 \%$ for two-way ANOVA and $1 \%$ for Multivariate Linear Regression tests, giving comparable threshold $P$ values. Both methods allowed us to reveal a synergistic effect of SAHA and MTR, when taking into account simultaneously their individual effect and thus their additive effect. Functional annotation of differentially expressed genes was done using DAVID Functional Annotation tool (Benjamini-Hochberg $P$ value $<0.05)[15,16]$. This tool was used to assess whether specific biological pathways and functional gene ontology terms were overrepresented among the differentially expressed genes and within specific gene clusters. Also, enriched biological pathways and functional gene ontology terms were identified after applying a modified Fisher exact test and a Benjamini-Hochberg multiple test correction.

\section{SAHA and MTR synergistic, potentiating, or antagonistic effects determination}


Synergistic, potentiating, or antagonistic effects were deduced from the values of fold changes (FC) according to the following pharmacological standards: additive effects for FCSAHA + MTR = FCSAHA + FCMTR; synergistic effects for FCSAHA:MTR > FCSAHA + FCMTR; potentiation by MTR for FCSAHA:MTR > FCSAHA with no effect of MTR alone; potentiation by SAHA for FCSAHA:MTR > FCMTR with no effect of SAHA alone; and antagonism for FCSAHA:MTR < FCSAHA plus FCMTR.

\section{Quantitative real-time polymerase chain reaction (RT-qPCR)}

Expression levels of 12 genes were analyzed using real-time quantitative PCR as a validation of microarray results. Reverse transcription was performed with $500 \mathrm{ng}$ of total RNA and 200 UI MMLV Reverse Transcriptase (Life Technologies, France) according to the manufacturer's instructions. PCRs were performed using TaqMan Gene Expression Assays (Life Technologies) for CAMK2N2 (Hs00536421_m1), DACT3 (Hs00376818_m1), FERMT3 (Hs01075695_m1), KDM4B (Hs00943639_m1), PREX1 (Hs0036807_m1), NCOR2 (Hs00196955_m1), GRK5 (Hs00992173_m1), BAMBI (Hs03044164_m1), DLK2 (Hs01106587_m1), NGFR (Hs00609977_m1), SLC4A11 (Hs00984689_m1), CORO1A (Hs00200039_m1), and TBP (Hs00427620_m1). All real-time PCRs were performed using the MX3000P detection system (Life Technologies) and the amplifications were done using the TaqMan Universal PCR Master Mix (Life Technologies). Experiments were carried out in duplicate for each data point. The relative quantification in gene expression was determined using the $2^{-\Delta \Delta \mathrm{Ct}}$ method [24] and fold changes (FC) in gene expression were normalized to $T B P$ internal control and relative to untreated control condition (calibrator).

\section{Results}

\section{Synergistic cell death induced by SAHA plus MTR combination in SS tumor cells}

The cell-death-inducing effects of single agent SAHA or MTR was studied in primary tumor cells from 4 SS patients (P1-P4) (characteristics in Supplementary Data, Table S1). Doseresponse survival curves to a 48-h treatment are shown in Fig. 1a, b. Cell death occurred mainly by apoptosis since, in all conditions, $>70 \%$ of the non-viable cells bound the apoptotic indicator annexin V (not shown). The sensitivity to the drugs was variable, cells from P3 and P4 being hardly and highly sensitive to MTR, respectively. Cells from the 4 patients were treated with serial dilutions of each drug individually and with both drugs simultaneously at a fixed ratio of doses that corresponded to $1 / 2,1,112$

\section{, 2, and 212}

of the individual ED50 obtained for P2 cells (i.e., $4.05 \mu \mathrm{M}$ and $328 \mathrm{nM}$ for SAHA and MTR, respectively) that display an average sensitivity (Fig. 1). Figure 2 shows fractional survival dose-response curves from at least three independent experiments. For P3 cells, the combined treatment overcame the insensitivity to MTR used as a single agent. The ED50 of each drug used in combination was extrapolated from the equation of the combined response curves and corresponded to 4/9, 2/3,3/4, and 1/3 of the ED50 of the drugs used in mono-treatments for P1, P2, P3, and P4, respectively. As shown in Table 1 and Supplementary Data, Figure S1, SAHA and MTR displayed a clear synergistic behavior for cells of 4 patients indicated by CI values below 1. DRI values of SAHA and MTR, which are a useful indicator of potential clinical benefit (Supplementary Data, Table S2), were much greater than 1 for all the patients 
at doses affecting over $80 \%$ of the cells, reflecting a sharp decrease in combined treatment doses required compared to single treatments. The extreme positive values of DRIs obtained for MTR-treated P3 cells reflect their insensitivity to the drug when used alone.
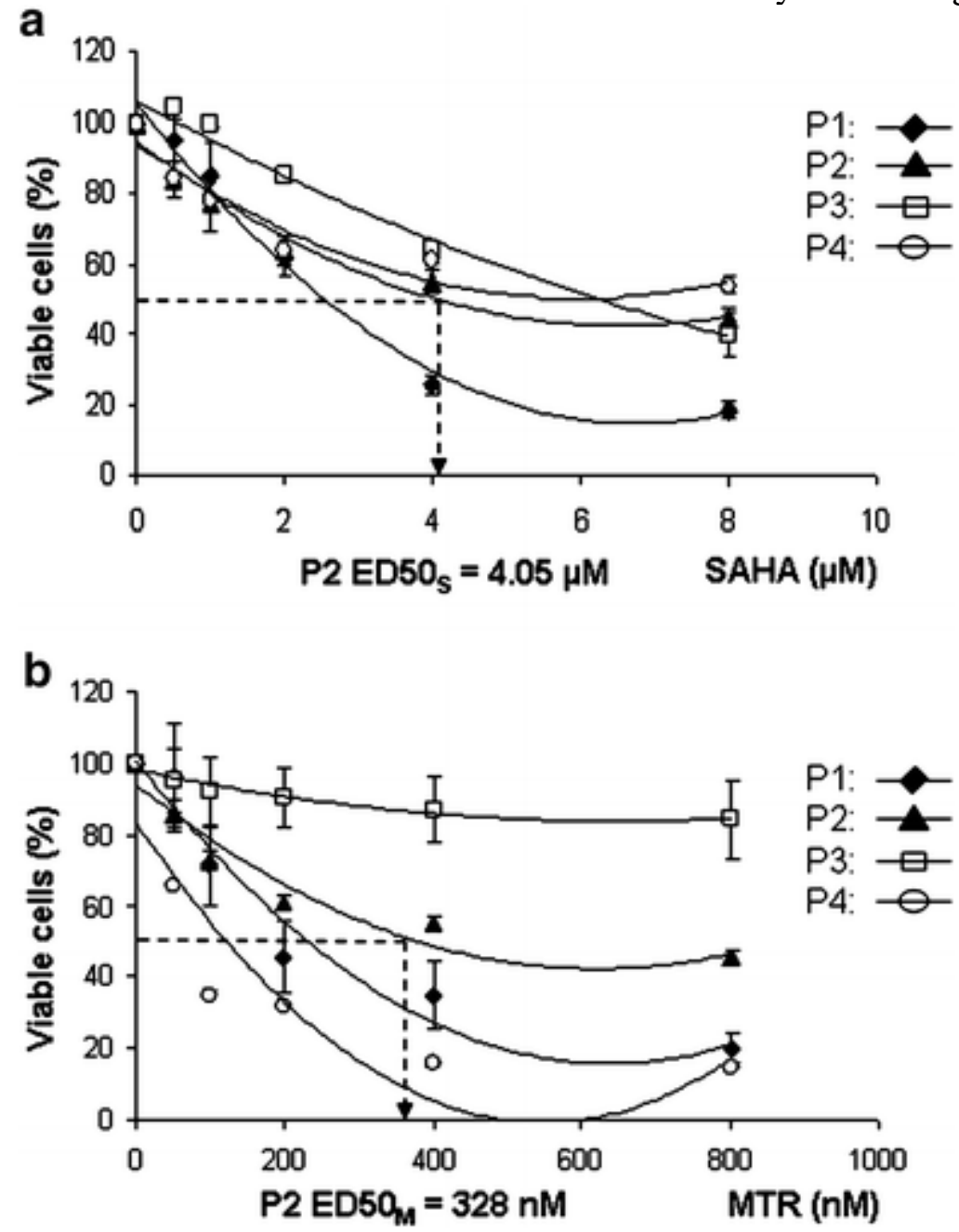

Fig. 1

Effect of SAHA (a) or MTR (b) on the survival of primary SS cells. Cells from patients 1-4 (P1-P4) that survived after a 48-h treatment were identified by FACS analysis as annexin V and 7-AAD negative (viable). They were quantified as percent of total gated parental cells normalized to that in control untreated populations. Values are means of three independent biological replicates. Order two polynomial trend lines were chosen for graphical representation and efficient dose 50 (ED50) values were derived from the corresponding equations. ED50 values are indicated for P2 who responds with an average sensitivity to both drugs 

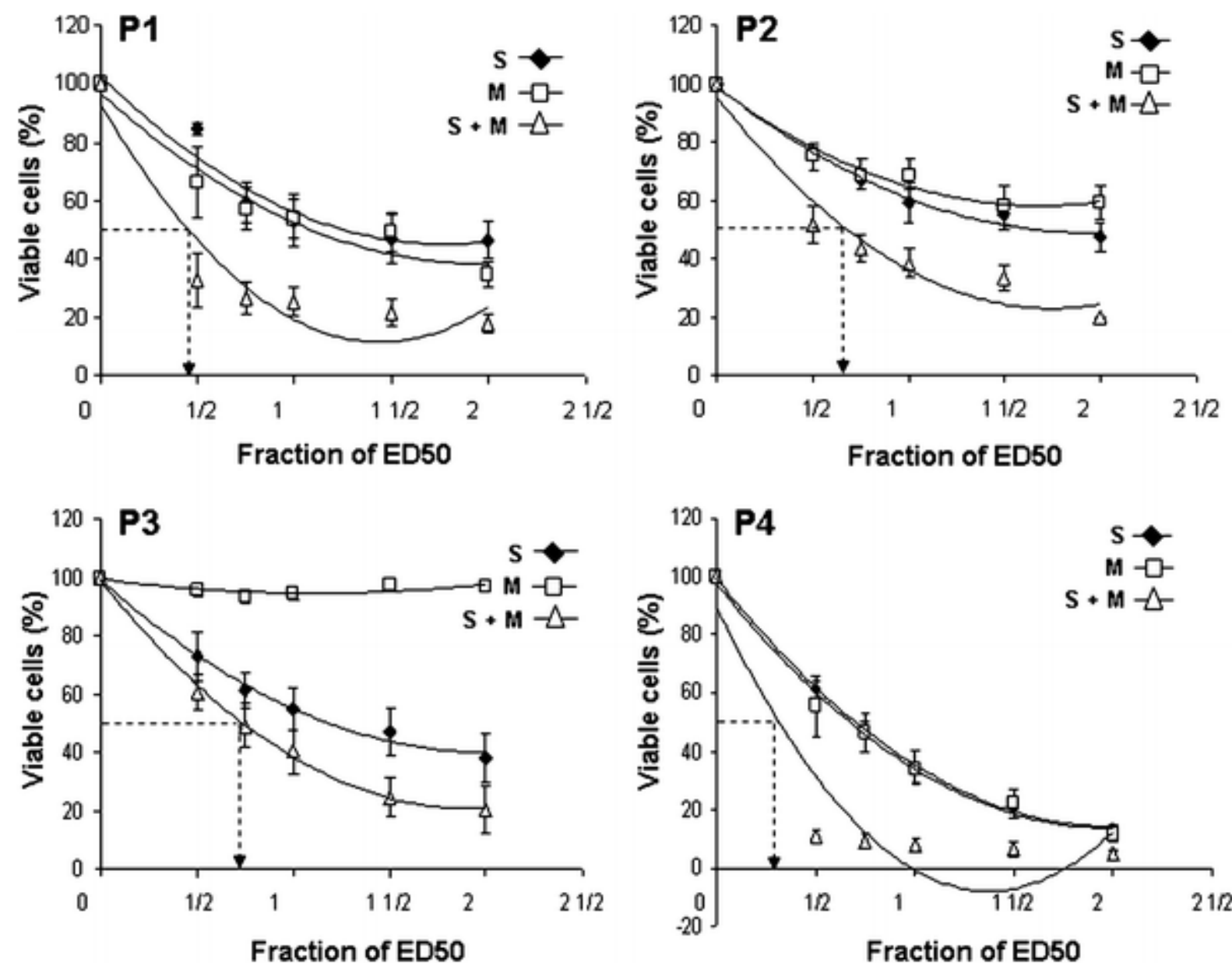

Fig. 2

Effect of SAHA plus MTR treatment on the survival of primary SS cells. Drug combination effect was determined according to the design of Chou and Talalay [7]. Cascade fractions of the ED50 of each drug deduced for P2 (i.e., $4.05 \mu \mathrm{M}$ and $328 \mathrm{nM}$ for SAHA and MTR, respectively) were applied to the 4 patients in mono- or combined treatments. Cell survival was assessed, quantified, and represented as for Fig. 1. Values are means of three independent biological replicates. The ED50 of each drug used in combination was extrapolated from the equations of the combined response curves and corresponded to 4/9,2/3, 3/4 and 1/3 of the ED50 of the drugs used in mono-treatments for P1, P2, P3, and P4, respectively (see dotted arrows)

Table 1

CI at combined (1:1) doses of SAHA and MTR corresponding to serial dilutions of their ED50

\section{Fraction ED50}

\section{$\begin{array}{lllll}0.5 & 0.75 & 1 & 1.5 & 2\end{array}$}

P1

$\begin{array}{llllll}\mathrm{Fa}^{\mathrm{a}} & 0.72 & 0.78 & 0.78 & 0.81 & 0.75 \\ \text { CI values }^{\mathrm{b}} & 0.030 & 0.013 & 0.000 & 0.009 & 0.004\end{array}$

Graded symbols ${ }^{\mathrm{c}}+++++++++++++++++++++++++$ 


\section{Fraction ED50}

\section{$\begin{array}{lllll}0.5 & 0.75 & 1 & 1.5 & 2\end{array}$}

P2

$\begin{array}{llllll}\mathrm{Fa} & 0.77 & 0.80 & 0.83 & 0.85 & 0.91 \\ \text { CI values } & 0.078 & 0.009 & 0.002 & 0.001 & 0.000\end{array}$

Graded symbols +++++++++++++++++++++++++

P3

$\begin{array}{llllll}\text { Fa } & 0.53 & 0.62 & 0.69 & 0.81 & 0.84 \\ \text { CI values } & 1.478 & 0.319 & 0.080 & 0.003 & 0.001 \\ \text { Graded symbols } & - & +++ & +++++ & +++++ & +++++ \\ \text { P4 } & & & & & \\ \text { Fa } & 0.97 & 0.98 & 0.98 & 0.99 & 0.99 \\ \text { CI values } & 0.004 & 0.001 & 0.001 & 0.000 & 0.000 \\ \text { Graded symbols } & \text { +++++ } & \text { +++++ } & \text { +++++ }\end{array}$

${ }^{a} \mathrm{Fa}$ is the fraction of cells affected, i.e., in this experiment, the proportion of dead cells among the total treated cells

${ }^{\mathrm{b}} \mathrm{CI}$ is the Combination Index that measures the degree of drug interaction in terms of additivity $(\mathrm{CI}=1)$, synergism $(\mathrm{CI}<1)$, or antagonism $(\mathrm{CI}>1)$ for a given endpoint of the effect measurement

${ }^{c}$ The graded symbols indicate the following: +++++ very strong synergy $(<0.1)$; +++ synergism (0.1-0.3); and --- antagonism (1.45-3.3), according to the semiquantitative scale of Chou [7]. Values were obtained with the help of CalcuSyn software. P1-P4: patients 1-4

\section{Delineation and characteristics of subsets of genes differentially responsive to SAHA and/or MTR}

To identify genes whose expression was differentially altered by SAHA and/or MTR treatments, we performed a gene expression profiling in cell samples from the 4 patients. We treated these with doses of drugs corresponding to the ED50 obtained for the combined treatment of cells from P2, i.e., $2.7 \mu \mathrm{M}$ and $219 \mathrm{nM}$ for SAHA and MTR, respectively. Given the high death response of cells from patient 4 , the length of treatment was reduced to $24 \mathrm{~h}$ for all the patients. After multivariate analysis, genes whose expression was influenced by SAHA or MTR mono-treatments and SAHA plus MTR combined treatment are listed according to their response to the drugs and their up- or down-regulation in Supplementary Data, Table S3. Among a total of 2399 selected genes, the vast majority responded differentially to either SAHA or MTR mono-treatments in about equal proportion, i.e., 51.1\% for SAHA (1226 genes) and $45.4 \%$ for MTR (1090 genes), while only a few genes differentially responded to the combined treatment, i.e., 3.5\% (83 genes). In each of the three subsets, similar proportions of genes were up- and down-regulated, i.e., 46.4\% (569 genes) and 53.6\% (657 genes) for SAHA, 48.4\% (528 genes) and 51.6\% (562 genes) for MTR, and 59\% (49 genes) and 41\% (34 genes) for SAHA plus MTR, respectively. The genes were classified similarly for the 4 patients as illustrated by dendrograms and heat map representation of their hierarchical clustering (Figs. 3, 4). The two Venn diagram representations of the overlap between the three subsets of up- or down-regulated genes (Supplementary Data, Figure S2) show that most of 
the genes responding to either of mono-treatments did it exclusively, while a minor subset of genes responded independently to both drugs (noted as SAHA and MTR). Among the genes that differentially responded to the combined treatment (noted as SAHA:MTR), nearly half did so exclusively, while the remaining genes already responded to mono-treatments by SAHA and/or MTR. The lists of genes corresponding to the different subgroups are reported in Supplementary Data, Table S4. SAHA:MTR responsive genes were further classified according to the synergistic, potentiating, or antagonistic effects of the two drugs on their expression.

a

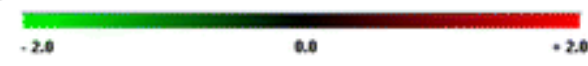

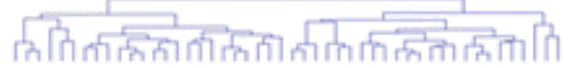

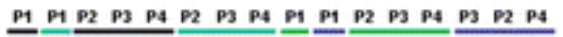

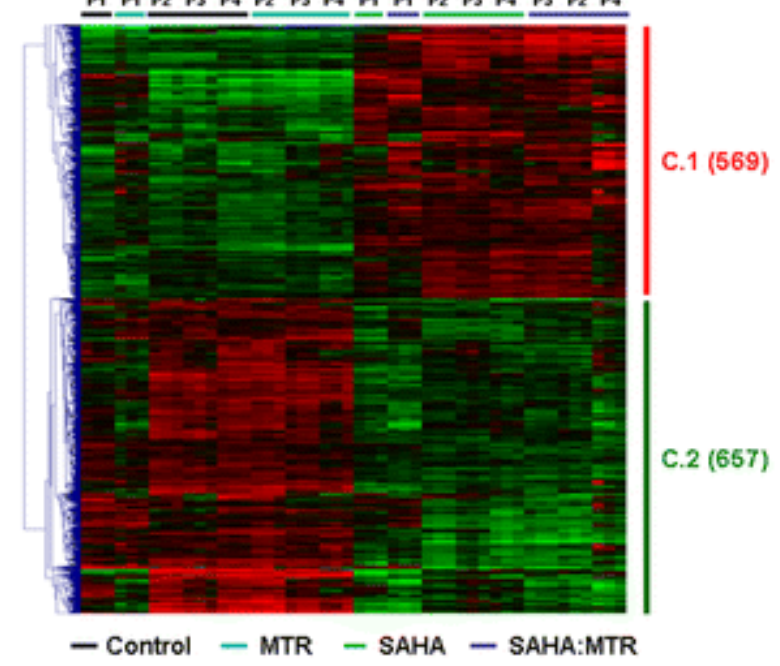

b
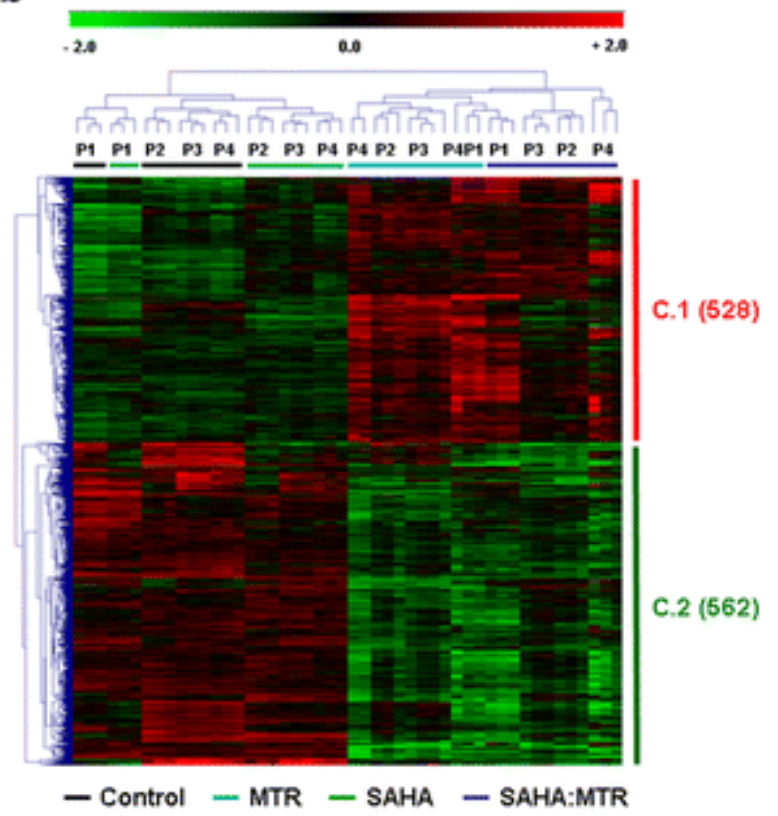

Fig. 3

Hierarchical clustering of the genes differentially responsive to mono-treatments.

Dendrograms of patients and gene clustering feature in columns and rows, respectively. According to a $\log 2$ pseudocolor scale, red indicates a high level of mRNA expression compared to the median value of a given gene, whereas green indicates a low level of expression. Differentially expressed genes were selected according to a filter criterion of more than a twofold change. a Heat map visualization of SAHA significant genes including up(Cluster 1) and down- (Cluster 2) regulated genes; based on gene expression, patients were clustered into two groups, i.e., SAHA- and NON-SAHA-treated. b Heat map visualization of MTR significant genes including up- (Cluster 1) and down- (Cluster 2) regulated genes; based on gene expression, patients were clustered into two groups, i.e., MTR- and NON-MTRtreated 


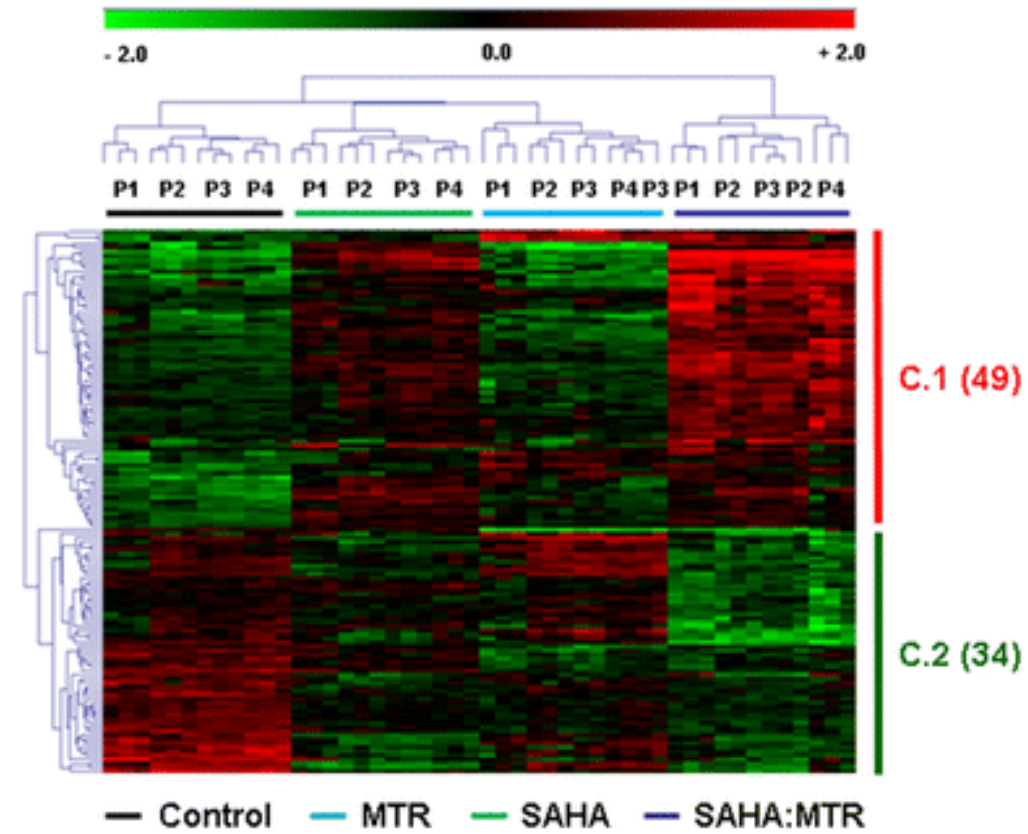

Fig. 4

Hierarchical clustering of the genes differentially responsive to combined treatments. Dendrograms of patients and gene clustering feature in columns and rows, respectively. Meaning of colors and selection of differentially expressed genes are detailed in Fig. 3. Heat map visualization of SAHA:MTR significant genes including up- (Cluster 1) and down(Cluster 2) regulated genes; based on gene expression, patients were clustered into two groups, i.e., SAHA + MTR- and NON- or MONO-treated

As shown in Supplementary Data, Table S5, the expression of a large proportion of SAHA:MTR responsive genes was modulated synergistically or potentiated by MTR, while that of smaller proportion was modulated antagonistically. The transcriptional response of representative genes belonging to each of the subsets delineated by the Venn diagram has been illustrated by box plots (Supplementary Data, Figure S3).

\section{Modulation of Sp factors' transcriptional activity by SAHA and/or MTR}

SAHA mediates some of its effect via the modulation of Sp1 (or Sp3) transcriptional activity and MTR is a DNA binding compound which can directly inhibit the binding of Sp factors to GC-rich promoter motifs. To evaluate to which extent those direct mechanisms might contribute to the expression of the genes responsive to SAHA:MTR, we screened these for enrichment of Sp1 DNA binding motifs in widening the screening to the sets of genes differentially responsive to SAHA or MTR mono-treatments (Supplementary Data, Table S3). We found that Sp1 motifs ranked first (corrected Benjamini $P$ value 3.50-18), for around a third of the genes up-regulated by SAHA (i.e., 191/528 genes). A similar result was obtained using DIstant Regulatory Elements (DIRE) search tool [14]. When cross-checking the latter subset of genes with the genes responsive to SAHA:MTR, we found a minor overlap of seven up-regulated genes, i.e., CDC42EP2, SH2B2, CNFN, DLK2, MAP1A, NGFR, and TNFRSF12A (Supplementary Data, Table S6) that might thus represent direct targets of Sp1 (or Sp3). Interestingly, except for SH2B2, all these genes belonged to the subset of genes potentiated by MTR (Supplementary Data, Table S5). No enrichment of Sp1 motifs was found for SAHA down-regulated genes and for MTR up- and down-regulated ones. 


\section{Most significant genes and processes differentially targeted by SAHA}

Gene ontology (GO) enrichment analysis identified nucleosome as the only significant term associated with SAHA up-regulated genes (Table 2). It encompasses 15 histone genes, among which H1F0 was first-ranked (Supplementary Data, Table S7). A thorough examination of the function of the most strongly up-regulated genes (Supplementary Data, Table S3) indicated that many of them were related to cancer and encoded various effectors of signal transduction, cell cycle, and transcriptional regulation (Table 3; Supplementary Data, Table S8 for details on their behavior). The highest FC (>13) were obtained for three tumor suppressors, i.e., CAMK2N2 (alias CAM-KIIN), DACT3 (alias DAPPER3), and CDKN1C (alias p57Kip2). Table 2

Most significant gene ontology terms and KEGG pathways found for SAHA- or MTRregulated gene

\begin{tabular}{|c|c|c|c|}
\hline \multicolumn{2}{|c|}{ Drug response } & GO terms and KEGG pathways ${ }^{a}$ & $P$ value $^{\mathrm{b}}$ \\
\hline & Up & Nucleosome & $9.04 \mathrm{E}-04$ \\
\hline & & GTPase regulator activity & $2.05 \mathrm{E}-05$ \\
\hline & & SH2 domain & $2.90 \mathrm{E}-05$ \\
\hline & & Leukocyte activation & $4.20 \mathrm{E}-05$ \\
\hline SAHA & Down & Regulation of apoptosis & $2.60 \mathrm{E}-03$ \\
\hline & Down & Pleckstrin homology & $2.60 \mathrm{E}-03$ \\
\hline & & Regulation of cytokine production & $1.40 \mathrm{E}-02$ \\
\hline & & IL-7 transduction & $3.60 \mathrm{E}-02$ \\
\hline & & NOD-like receptor signaling pathway & $1.60 \mathrm{E}-02$ \\
\hline & & Phosphorylation & $1.64 \mathrm{E}-34$ \\
\hline & & Acetylation & $1.10 \mathrm{E}-13$ \\
\hline & & ATP binding & $3.30 \mathrm{E}-10$ \\
\hline & & Alternative splicing & $1.60 \mathrm{E}-06$ \\
\hline & & SH3 domain & $3.20 \mathrm{E}-06$ \\
\hline & & Cytoskeleton & $3.40 \mathrm{E}-06$ \\
\hline & & GTPase regulator activity & $4.20 \mathrm{E}-06$ \\
\hline MTR & Down & Pleckstrin homology & $9.10 \mathrm{E}-05$ \\
\hline & & Src homology-3 domain & $1.10 \mathrm{E}-04$ \\
\hline & & Chromatin regulator & $2.10 \mathrm{E}-04$ \\
\hline & & WD repeat & $6.00 \mathrm{E}-04$ \\
\hline
\end{tabular}

Fc gamma R-mediated phagocytosis pathway $2.30 \mathrm{E}-05$

Chemokine signaling pathway

$1.50 \mathrm{E}-04$

Fc epsilon R1 signaling pathway

$5.70 \mathrm{E}-04$

Non-small cell lung cancer pathway

$1.60 \mathrm{E}-02$

\footnotetext{
${ }^{a}$ Obtained with DAVID Functional Annotation tool [11]

${ }^{\mathrm{b}}$ Corrected Benjamini-Hochberg $P$ value [11]
} 
Table 3

Most significant cancer-related genes up- and down-regulated by SAHA or MTR monotreatments

\begin{tabular}{|c|c|c|c|c|c|}
\hline Drug response & Gene symbol & $P$ value $^{b}$ & $\mathbf{F C}^{\mathbf{c}}$ & Sensitivity $^{d}$ & ${ }^{d}$ References $^{e}$ \\
\hline & CAMK2N2 & $4.60 \mathrm{E}-21$ & 14.67 & S & {$[1]$} \\
\hline & $C D C 42 E P 2$ & $7.27 \mathrm{E}-19$ & 3.03 & S, S:M & {$[2]$} \\
\hline & $H S D 17 B 8$ & $3.20 \mathrm{E}-17$ & 4.83 & S & {$[3]$} \\
\hline & $F G F R 3$ & $1.37 \mathrm{E}-16$ & 7.86 & S, S:M & {$[4]$} \\
\hline & $D A C T 3$ & $1.86 \mathrm{E}-15$ & 19.41 & S & {$[5]$} \\
\hline $\mathrm{Un}$ & $C D C 37$ & $2.15 \mathrm{E}-15$ & 2.68 & S & {$[6]$} \\
\hline up & GRHL1 & $1.48 \mathrm{E}-14$ & 3.13 & $\mathrm{~S}$ & [7] \\
\hline & $D L E U 1$ & $5.91 \mathrm{E}-14$ & 4.27 & S, S:M & {$[8]$} \\
\hline & MAPT & $1.86 \mathrm{E}-13$ & 9.54 & S & [9] \\
\hline & $C D K N 1 C$ & $1.15 \mathrm{E}-11$ & 13.59 & $\mathrm{~S}$ & {$[10]$} \\
\hline & CRIP2 & $2.04 \mathrm{E}-09$ & 7.25 & S & [11] \\
\hline SAHA & $J A G 2$ & $2.44 \mathrm{E}-09$ & 3.06 & $\mathrm{~S}$ & {$[12]$} \\
\hline ГАПA & FERMT3 & $4.61 \mathrm{E}-21$ & -8.45 & S, S:M & {$[13]$} \\
\hline & PTPN7 & $3.80 \mathrm{E}-18$ & -6.12 & S & [14] \\
\hline & $C D K L 1$ & $3.91 \mathrm{E}-18$ & -3.40 & $S$ & {$[15]$} \\
\hline & $R G L 4$ & $1.02 \mathrm{E}-17$ & -30.83 & S & {$[16]$} \\
\hline & DOK2 & $2.54 \mathrm{E}-15$ & -7.60 & S & [17] \\
\hline Down & PSMB10 & $2.56 \mathrm{E}-15$ & -6.31 & S & {$[18]$} \\
\hline Down & $I L 27 R A$ & $3.77 \mathrm{E}-15$ & -7.73 & S & [19] \\
\hline & FUT7 & $4.67 \mathrm{E}-15$ & -5.17 & $\mathrm{~S}, \mathrm{M}, \mathrm{S}: \mathrm{M}$ & {$[20]$} \\
\hline & MGAT3 & $8.91 \mathrm{E}-15$ & -2.83 & S, M, S:M & [21] \\
\hline & PTPRCAP & $1.11 \mathrm{E}-14$ & -8.82 & S & {$[22]$} \\
\hline & $F M N L 1$ & $5.23 \mathrm{E}-14$ & -4.74 & S & {$[23]$} \\
\hline & ICAM3 & $3.76 \mathrm{E}-13$ & -4.43 & S & {$[24]$} \\
\hline & $B R D 8$ & $2.19-16$ & 2.95 & M & [25] \\
\hline & $T O B 1$ & $8.78 \mathrm{E}-14$ & 2.61 & M & [26] \\
\hline $\mathrm{Un}$ & SNHG1 & $1.29 \mathrm{E}-13$ & 3.10 & M & [27] \\
\hline op & BIRC2 & $3.66 \mathrm{E}-11$ & 2.30 & M & [28] \\
\hline & $I S C U$ & $1.22 \mathrm{E}-08$ & 2.61 & M, S:M & [29] \\
\hline & C22orf29 & $3.41 \mathrm{E}-08$ & 2.96 & M & [30] \\
\hline 101 & $K D M 4 B$ & $2.92 \mathrm{E}-26$ & -10.48 & M & [31] \\
\hline & CHST11 & $2.62 \mathrm{E}-22$ & -5.49 & M & [32] \\
\hline Dous & PREX1 & $1.14 \mathrm{E}-20$ & -6.77 & M & [33] \\
\hline DOWn & NCOR2 & $9.17 \mathrm{E}-20$ & -13.71 & M & [34] \\
\hline & CPPEDI & $1.02 \mathrm{E}-19$ & -3.59 & M, S:M & [35] \\
\hline & $A T P 11 A$ & $7.00 \mathrm{E}-19$ & -4.05 & M & [36] \\
\hline
\end{tabular}




$\begin{array}{ccccc}\text { Drug response Gene symbol } & \boldsymbol{P}_{\text {value }}^{\mathbf{b}} & \text { FC }^{\mathbf{c}} & \text { Sensitivityd }^{\mathbf{d}} \text { References }^{\mathbf{e}} \\ \text { MADIL1 } & 4.07 \mathrm{E}-18-6.85 & \mathrm{~S}, \mathrm{M} & {[37]} \\ \text { TBC1D6 } & 1.78 \mathrm{E}-17-3.26 & \mathrm{M} & {[38]} \\ D N M 2 & 1.24 \mathrm{E}-16-4.35 & \mathrm{M} & {[39]} \\ C A M K 1 D & 2.92 \mathrm{E}-16-5.79 & \mathrm{M} & {[40]} \\ B R E & 3.98 \mathrm{E}-15-3.06 & \mathrm{M} & {[41]} \\ C M I P & 7.72 \mathrm{E}-15-5.09 & \mathrm{M} & {[42]}\end{array}$

${ }^{a}$ Only the 12 most significant cancer-related genes are listed for SAHA up- or down- and MTR down-regulated genes

${ }^{\mathrm{b}}$ Nominal $P$ value

${ }^{\mathrm{c}}$ Fold changes (FC) are expressed relative to untreated cell samples

${ }^{\mathrm{d}} \mathrm{S} ; \mathrm{M} ; \mathrm{S}, \mathrm{M}$; and S:M refer to differential responses to SAHA, MTR, SAHA and MTR, and SAHA:MTR interaction

${ }^{\mathrm{e}}$ References are listed in supplementary Data, Table S8

GO enrichment analysis on genes down-regulated in response to SAHA showed that they belonged to several biological processes and pathways altered by the drug in SS cells (Table 2; Supplementary Data, Table S7 for details on genes). The drug decreased the expression of many major positive regulators and effectors of leukocyte activation such as CD3D, CD2, LCP2, LAT, LCK, and ZAP70. We also found that it decreased the expression of DOK2, PSTPIP1 (alias CD2BP1), MAP4K1 (alias HPK1), and PTPN7 (alias HePTP) involved in negative feedback loops. Several members of the Janus kinase/Signal Transducer and Activator of Transcription (JAK/STAT) signaling pathway were also decreased such as JAK3, STAT6, STAT5A, STAT5B, NMI, IL2RG, and IL27RA, as well as several critical negative regulators of apoptosis like IRAK1, CARD8 (alias CARDINAL or TUCAN), AVEN, TNFSF12 (alias TWEAK), CASP4, FAIM, and CASP5 (Supplementary Data, Table S7). The collapse of pro-inflammatory pathways was evidenced by the decreased expression of TLR1, MYD88, PYDC1, PYCARD (alias ASC), CARD6, TRIP6, XIAP, HDAC7, and MAPK13 (alias p38delta). Finally, more genes, unclassified in GO terms and known as related to cancer, also figured among the most significant down-regulated genes, as PTPN7 (alias HePTP), PTPRCAP (alias CD-45-AP), FUT7, FMNL1, ICAM3, CDKL1, and PSMB10 (alias LMP10) (Table 3; Supplementary Data, Table S8 for details on the top-ranked genes).

\section{Most significant genes and cell processes targeted by MTR}

GO enrichment analysis did not identify significant term for MTR up-regulated genes. Markedly, a high proportion of the latter (110/528, i.e., around $21 \%$ ) encoded long noncoding RNAs (LncRNAS), while this proportion was much lower among the down-regulated genes (15/562, i.e., around $2.7 \%)$, or among any other cluster of drug-responsive genes (Supplementary Data, Table S3). Of note too, SNHG1, which encodes small nucleolar RNAs (snoRNA) described as diagnostic and prognostic marker in peripheral T cell lymphoma, was among the top-ranked genes up-regulated by MTR (Table 3; Supplementary Data, Table S8 for details on the genes). 
MTR down-regulated genes were classified into many significant GO terms and KEGG pathways (Table 2; Supplementary Data, Table S7). As for SAHA targets, "GTPase regulator activity" and "pleckstrin homology" were enriched. The great majority of the genes included in the above two GO terms were exclusive targets of each drug (Table S7). MTR selectively down-regulated genes encoding GEFs (PREX1, ELMO1, RAPGEF1, TRIO, and VAV2) and GAPs (MYO9B, SIPA1L1, RASA3, and ASAP1), the Rho GTPase ARHGAP26, the G-coupled protein kinase $A D R B K 2$ (alias GRK3), the intracellular trafficking regulator DNM2 (alias $D Y N 2$ ), the actin binding protein $S W A P 70$, the oncogenic kinase $M A P 4 K 4$, the T cell motility regulator $S K A P 1$ (alias $S K A P 55$ ), and the autophagy regulator $T B C 1 D 5$. Additional targets of MTR connected to cell motility, adhesion, and invasiveness were found in other related GO terms and KEGG pathways, like GRK5, GRK6, CHST11, CAMK1D, CASK, ACTR3, DISC1, FUT8, FNBP1, BOP1, PAK1, CCR1, and CXCR3 (Table S7). The drug also decreased the expression of many genes encoding positive effectors of proliferative and survival pathways like $B L K$ and PLCG2 (BCR signaling), LRBA (EGFR signaling), RPTOR, GNB1, PRKCA and $P R K C B$ (mTOR signaling), and TBLIX ( $\beta$-catenin and NF- $\kappa \mathrm{B}$ transcriptional activity), as well as GRB2, GRAP, GRAP2, and SH2B3 encoding signal adaptors. Moreover, MTR downregulated a number of members of the mitogen-activated protein (MAP) kinase signaling cascades as MAP2K5, MAP3K5, MAP4K4, MAPK1, MAP2K2, and MAP2K3 (Table 3; Supplementary Data, Table S8).

\section{Most significant genes and cell processes targeted by SAHA plus MTR combined treatment}

We examined the function of the genes whose expression was either synergized or potentiated (Supplementary Data, Table S5). Many of these genes were implicated in cancer and/or T cell functions (Table 4; Supplementary Data, Table S9 for details). Several tumor suppressors figured out among the up-regulated ones, as EIF4EBP1 (alias 4E-BP1), NPTX1, DLK2, $N G F R$ (alias $p 75 N T R$ ), CDC42EP2 (alias BORG1), and BIK. The up-regulation of some oncogenes was found too, as FGF22, FGFR3, SLC22A17, TNFRSF12A (alias FN14), and $M A P 1 A$. Some oncogenes implicated in signal transduction were among the down-regulated genes, as FBLN5 (alias DANCE), B3GALT4, and PPP1R10 (alias PNUTS). The expression of three genes encoding transcriptional regulators was also decreased, namely TADA3 (alias $h A D A 3), P B X 2$, and $A D A R$. SAHA:MTR interaction also induced a diminution in the expression of two members of the antigen processing and presenting machinery (APM) of class I molecules, i.e., TAP1 (alias ABCB2) and PSMB8 (alias LMP7). Finally, the examination of the set of genes whose expression was antagonized by the combined treatment (Table 4; Supplementary Data, Table S9) indicated that, for the vast majority of them, the upward or downward trends of their response to single treatments was not inverted by SAHA:MTR interaction (Supplementary Data, Table S5).

Table 4

Most significant cancer-related genes up- or down-regulated by SAHA:MTR interaction

\begin{tabular}{|c|c|c|c|c|c|}
\hline Drug response & Gene symbol & $P$ value $^{\mathbf{a}}$ FC $^{\mathrm{b}}$ & Sensitivity $^{c}$ & Behavior $^{d}$ & References $^{\mathbf{e}}$ \\
\hline \multirow{5}{*}{ Up } & $F G F 22$ & $7.42 \mathrm{E}-074.36$ & S:M & & {$[1]$} \\
\hline & EIF4EBP1 & $4.16 \mathrm{E}-052.18$ & $\mathrm{~S}: \mathrm{M}$ & & {$[2]$} \\
\hline & SLC22A17 & $1.34 \mathrm{E}-044.11$ & S:M & Synergy & {$[3]$} \\
\hline & NPTX1 & $6.44 \mathrm{E}-044.71$ & S:M & & {$[4]$} \\
\hline & $B I K$ & $9.47 \mathrm{E}-042.03$ & S:M & & [5] \\
\hline
\end{tabular}




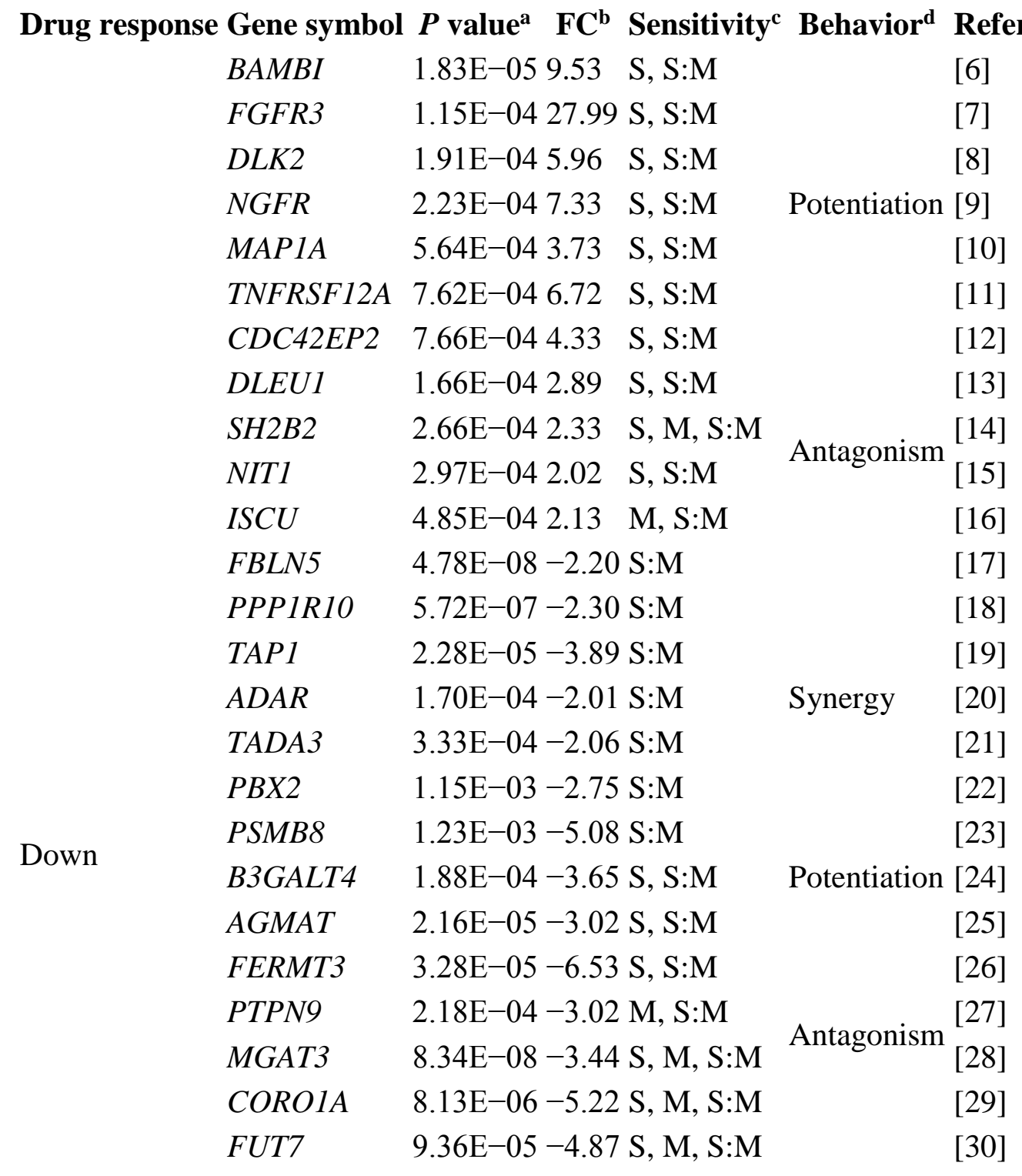

a, b, c As for Table 3

${ }^{\mathrm{d}}$ Synergy refers to the behavior of genes which only responded to SAHA:MTR interaction; potentiation refers to the behavior of genes whose response to SAHA was amplified by SAHA:MTR interaction; antagonism refers to the behavior of genes whose response to SAHA and/or MTR was reduced by SAHA:MTR interaction (refer to Table S5 for details)

${ }^{\mathrm{e}}$ References are listed in Supplementary Data, Table S9

\section{Validation of expression profiles of selected genes by quantitative real-time polymerase chain reaction}

To verify the accuracy of changes in gene expression deduced from the above transcriptomic analyses, selected genes were assayed by RT-qPCR from relevant mRNA extracts used for the microarrays. These corresponded to cells from 3 of the 4 patients, three biological replicates, and 4 treatment conditions, i.e., no drug for all the genes; SAHA for CAMK2N2, DACT3, and FERMT3; MTR for KDM4B, PREX1, NCOR2, and GRK5; and SAHA plus 
MTR for BAMBI, DLK2, NGFR SLC4A11, and CORO1A. We found that the direction of changes obtained by RT-qPCR (Table 5) was in agreement with that deduced from the arrays for all the genes (Supplementary Data, Table S3).

Table 5

Real-time quantitative PCR of selected genes

\begin{tabular}{lcccc}
\multicolumn{1}{c}{ Genes } & Patient 1 & \multicolumn{1}{c}{ Patient 2 } & Patient 3 & Drugs \\
CAMK2M2 & $5.833 \pm 0.341$ & $54.300 \pm 8.132$ & $32.767 \pm 1.527$ & \\
DACT3 & $4.333 \pm 0.425$ & $140.000 \pm 22.910$ & $31.167 \pm 3.694$ & SAHA \\
FERMT3 & $0.122 \pm 0.021$ & $0.073 \pm 0.002$ & $0.080 \pm 0.010$ & \\
KDM4 & $0.009 \pm 0.001$ & $0.029 \pm 0.008$ & $0.147 \pm 0.003$ & \\
PREX1 & $0.092 \pm 0.007$ & $0.196 \pm 0.047$ & $0.148 \pm 0.027$ & MTR \\
NCOR2 & $0.128 \pm 0.004$ & $0.091 \pm 0.012$ & $0.088 \pm 0.014$ & \\
GRK5 & $0.055 \pm 0.004$ & $0.066 \pm 0.015$ & $0.022 \pm 0.004$ & \\
BAMBI & $26.633 \pm 7.89362 .650 \pm 13.400$ & $30.633 \pm 5.624$ & \\
DLK2 & $1.767 \pm 0.357$ & $8.850 \pm 0.884$ & $3.400 \pm 0.984$ & \\
NGFR & $28.067 \pm 5.960$ & $228.900 \pm 54.942$ & $66.567 \pm 11.745$ & SAHA:MTR \\
SLC4A11 & $16.367 \pm 0.708$ & $45.750 \pm 0.318$ & $29.033 \pm 3.462$ & \\
CORO1A & $0.124 \pm 0.011$ & $0.110 \pm 0.002$ & $0.048 \pm 0.008$ &
\end{tabular}

The relative quantification in gene expression was performed as detailed in "Methods"; values are means of biological triplicates \pm Standard Error of the Mean and correspond to fold change in gene expression relative to untreated condition

\section{Discussion}

Extensive transcriptional modifications rather than gene mutations seem to account for the cellular deregulations that cause the abnormal immunological behavior, the high capacity for survival and resistance to apoptosis, and the skin homing and migratory properties of SS cells. We thus tested the ex vivo killing efficiency of the association of SAHA and MTR that broadly targets transcription. Importantly, we found that this drug combination had a synergistic or potentiating effect on the killing of primary cells from 4 SS patients. Our transcriptomic analysis of the differential responses of genes to mono- or combined treatments showed highly significant changes of expression that provided putative clues on some transcriptional mechanisms that may underlie the combined effects of SAHA plus MTR, as well as on some key deregulated pathways targeted by the drugs.

A minor subset of seven genes differentially responsive to SAHA:MTR interaction was enriched in Sp1 regulatory sequences. Markedly, the expression of six of these genes (CDC42EP2, CNFN, DLK2, MAP1A, NGFR, and TNFRSF12A) was potentiated by MTR, suggesting that the two drugs cooperate to release a repressive binding of $\mathrm{Sp} 1$ (or Sp3). In favor of this, functional $\mathrm{Sp} 1$ binding sites were previously identified for the majority of the latter genes [18, 26, 29, 31] and an Sp1-containing repression complex recruiting HDAC1 was detected on the NGFR promoter [18]. However, an overall limited direct role for Sp1 (or Sp3) should be envisaged in the mechanism of interaction of the two drugs, given the lack of global enrichment for Sp1 motifs among SAHA down-regulated genes and among total MTR- 
regulated ones. This is not unexpected considering the many acetylated targets of SAHA and the fact that MTR, although commonly viewed as an inhibitor of Sp1, also behaves as a broader epigenetic drug by directly binding to histones, thus inducing deleterious epigenetic modifications [25]. Our data indicate that MTR down-regulated the expression of many genes playing a key role in epigenetic regulation as DNMT1, several genes encoding transcription cofactors implicated in histone modifications (histone-lysine demethylases and methyltransferases, and histone deacetylase), in ATP-dependent chromatin remodeling, and in gene co-activation and co-repression. Thus, similar to HDAC inhibitors, MTR may indirectly induce broad alterations of gene expression. For example, DNMT1 suppression should induce the re-expression of a large set of transcripts under control of DNA methylation, ranging from coding genes to microRNAs and LncRNAs non-coding RNA. MTR selectively up-regulated a large proportion of LncRNAS, a striking observation in view of the recent identification of Sézary cell-associated LncRNAs [23]. In light of the several reports showing crosstalk between histone deacetylases and lysine-specific demethylases or DNA methyl transferases, SAHA and MTR may exert a synergistic activity in part via their epigenetic-targeted modifications.

As summarized in Fig. 5, SAHA and MTR as a combination therapy may be an interesting therapeutic option in the treatment of CTCL. Such association should, in theory, be an effective therapeutic against the main pathways involved in carcinogenesis and tumor progression of the CTCL.

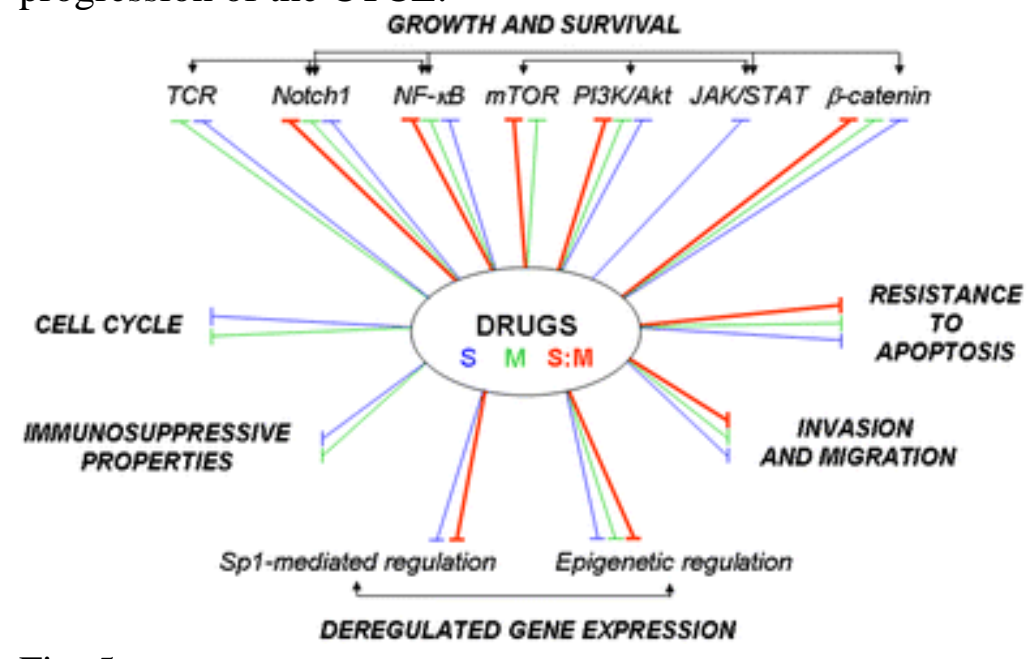

Fig. 5

Schematic representation of the major oncogenic signaling pathways and cellular mechanisms counteracted by SAHA and/or MTR treatments. Black arrows indicate possible crosstalk

\section{Counteraction of intertwined growth and survival pathways in SS cells}

Basal activation of the JAK/STAT pathway involved in IL-2 receptor (IL-2R) signaling and constitutive phosphorylations of STAT5 and STAT3 transcription factors are reported in CTCL lines [34]. In addition, STAT proteins are frequently overexpressed in Sézary patient cells due to a duplication at 17q11.2 [1]. SAHA repressed the expression of many genes coding for direct effectors of JAK-STAT signaling. The drug did not however act on the transcription of STAT3, or on that of its partner JAK2, but our data suggest that it could, together with MTR, counteract some of the constitutive phosphorylations of STAT3 via the inhibition of Notch1 and PI3K/Akt/mTOR pathways [2]. 


\section{Overtaking of the resistance of SS cells to apoptosis}

Low proliferative potential and accumulation of SS cells are likely to result from a defective regulation of intrinsic [34] and extrinsic [8,33] apoptosis pathways. Our findings strengthen the notion of a major impact of SAHA on the intrinsic death pathway [32] with the yet undescribed down-regulation of AVEN and CARD8 (alias TUCAN or CARDINAL) encoding, respectively, direct inhibitors of the formation and of the activity of the apoptosome, of XIAP (alias BIRC4) encoding a member of the Inhibitor of Apoptosis (IAP) family that inhibits caspase 9 activity, of FAIM encoding an inhibitor of XIAP ubiquitination and degradation, as well as the up-regulation of CRIP2 encoding a putative transcriptional activator of CASP3 and CASP9. SAHA may also activate the extrinsic pathway by decreasing the expression of TRIP6 encoding an antagonist of Fas/CD95-induced apoptosis.

\section{Neutralization of adhesion, invasion, and migration of SS cells}

An abnormally high expression of several chemokine receptors and of the adhesion molecule L-selectin (CD62L) was found in the majority of SS cells correlating with their central memory cell origin and with epidermotropism and lymphadenopathy [4, 6]. Markedly, MTR decreased CCR1, SAHA decreased SELL (CD62L), and both drugs independently decreased CXCR3. MTR should thus impede SS cell skin homing [27] by decreasing the expression of the three critical Rac activators: PREX1 (alias P-Rex1), DOCK2, and ELMO1 that transduce CXCL12-CXCR4 signaling. The overexpression of $\beta 1$ integrin $\mathrm{T}$ cell homing protein also has a high predictive power to classify SS patients [19] and was potently inhibited by SAHA plus MTR treatment. Our data strongly suggest that SAHA and MTR combination may counteract the invasive and migratory behavior of SS cells.

\section{Abolition of immunoregulatory properties of SS cells}

SS cells from some patients show features of Th2 and Tregs T lymphocytes in secreting IL-4, IL-5, IL-10, and TGF- $\beta$ immunosuppressive cytokines and expressing FOXP3 transcription factor, likely contributing to suppression of the host cellular immunity [20]. Given that the expression of IL-10, TGF- $\beta$, and FOXP3 relies on JAK/STAT signaling, treating SS cells with SAHA that profoundly alters this pathway should counteract some of SS cells' suppressive functions.

In conclusion, our study provides mechanistic clues on the individual and combined actions of SAHA and MTR in SS cells, which seems to rely on complementary or synergistic hampering of most of the survival and apoptotic processes currently known to be deregulated in these tumors. Gene targets of presumed importance were identified and deserve future functional validations. Some of these suggest in turn novel basal deregulations of SS cells and, as such, might in the future provide new markers for diagnosis and prognosis. Our results also emphasize the therapeutic interest of combining two epigenetic drugs which are probably interacting largely via additive or synergistic DNA and histone modifications. Lastly, they also point at some possibly detrimental side effects of this drug combination such as the weakening of tumor immunogenicity.

\section{Notes}

\section{Acknowledgements}


This work was supported by the Institut National de la Santé et de la Recherche Médicale (INSERM) and the French National Research Agency (ANR-08-SYSC-003 CALAMAR). We thank Ms. L. Borg for expert supervision of Marseille Luminy cell culture facilities, Dr. J. Imbert (TAGC), Dr. A. Bergon, and Mr. Nicolas Fernandez (transcriptomic and Genomic Marseille-Luminy TGML/TAGC platform) for their helpful advice for the preparation of the manuscript.

\section{Availability of supporting data}

The microarray data included in the paper have been deposited under embargo in GEO to be released upon acceptance of the paper for publication at: (http://www-ncbi-nlm-nihgov.gate2.inist.fr/geo/query/acc.cgi?token=glypyyownjmtnmp\&acc=GSE64119).

\section{Author contribution}

BKP and RTC initiated the Sézary project and RTC supervised hospital collaborations. PP supplied characterized PBMC samples from SS patients. BKP conceived the experiments; BKP, BL, JG, and NB (Beaufils) designed the experiments; and BKP, BL, NB (Beaufils), NB (Bonnet), RC, and TLT carried out the experiments. PR designed the statistical analyses of microarray data and RR carried out the bioinformatic analyses. BKP carried out the interpretation of the analyses and drafted the article. PR, RR, VG, and RTC corrected the manuscript. All authors read and approved the final manuscript.

\section{Compliance with ethical standards}

\section{Conflict of interest}

All the authors have no conflicts of interest to declare.

\section{Consent}

Patients have signed written informed consent before Sézary cell collection.

\section{Ethical approval}

The study was approved by the "Comite de Protection des Personnes" (CPP) SudMéditerranée II (ethics committee).

\section{Funding}

All the authors have no funding to declare.

\section{References}

1. Barba G, Matteucci C, Girolomoni G et al (2008) Comparative genomic hybridization identifies 17q11.2 approximately q12 duplication as an early 
event in cutaneous T-cell lymphomas. Cancer Genet Cytogenet 184:48-51. doi: 2 .

2. Bellei B, Pacchiarotti A, Perez M, Faraggiana T (2004) Frequent beta-catenin overexpression without exon 3 mutation in cutaneous lymphomas. Mod Pathol 17(10):1275-12813.

3. Blume SW, Snyder RC, Ray R et al (1991) Mithramycin inhibits SP1 binding and selectively inhibits transcriptional activity of the dihydrofolate reductase gene in vitro and in vivo. J Clin Investig 88:1613-1621. 4.

4. Campbell JJ, Clark RA, Watanabe R, Kupper TS (2010) Sezary syndrome and mycosis fungoides arise from distinct T-cell subsets: a biologic rationale for their distinct clinical behaviors. Blood 116:767-771. 5.

5. Campo E, Swerdlow SH, Harris NL et al (2011) The 2008 WHO classification of lymphoid neoplasms and beyond: evolving concepts and practical applications. Blood 117:5019-5032.

6. Capriotti E, Vonderheid EC, Thoburn CJ et al (2007) Chemokine receptor expression by leukemic $\mathrm{T}$ cells of cutaneous T-cell lymphoma: clinical and histopathological correlations. J Investig Dermatol 127:2882-2892. 7.

7. Chou T-C (2006) Theoretical basis, experimental design, and computerized simulation of synergism and antagonism in drug combination studies. Pharmacol Rev 58:621-681.

8. Contassot E, Kerl K, Roques S et al (2008) Resistance to FasL and tumor necrosis factor-related apoptosis-inducing ligand-mediated apoptosis in Sezary syndrome T-cells associated with impaired death receptor and FLICEinhibitory protein expression. Blood 111:4780-47879.

9. Costello R, Sanchez C, Le Treut T et al (2010) Peripheral T-cell lymphoma gene expression profiling and potential therapeutic exploitations. $\mathrm{Br} \mathrm{J}$ Haematol 150:21-27.

10. Dalloul A, Laroche L, Bagot M et al (1992) Interleukin-7 is a growth factor for Sézary lymphoma cells. J Clin Investig 90:1054-1060. 11.

11. Didier G, Brézellec P, Remy E, Hénaut A (2002) GeneANOVA—gene expression analysis of variance. Bioinform Oxf Engl 18:490-49112.

12. Duvic M, Vu J (2007) Vorinostat: a new oral histone deacetylase inhibitor approved for cutaneous T-cell lymphoma. Expert Opin Investig Drugs 16:1111-1120.

13. Gardner JM, Introcaso CE, Nasta SD et al (2009) A novel regimen of vorinostat with interferon gamma for refractory Sézary syndrome. J Am Acad Dermatol 61:112-116. 14.

14. Gotea V, Ovcharenko I (2008) DiRE: identifying distant regulatory elements of co-expressed genes. Nucleic Acids Res 36:W133-W139.

15. Huang DW, Sherman BT, Lempicki RA (2009) Bioinformatics enrichment tools: paths toward the comprehensive functional analysis of large gene lists. Nucleic Acids Res 37:1-13. 16.

16. Huang DW, Sherman BT, Lempicki RA (2009) Systematic and integrative analysis of large gene lists using DAVID bioinformatics resources. Nat Protoc 4:44-57.

17. Huang L, Sowa Y, Sakai T, Pardee AB (2000) Activation of the p21WAF1/CIP1 promoter independent of p53 by the histone deacetylase inhibitor suberoylanilide hydroxamic acid (SAHA) through the Sp1 sites. Oncogene 19:5712-5719. 
18. Iraci N, Diolaiti D, Papa A et al (2011) A SP1/MIZ1/MYCN repression complex recruits HDAC1 at the TRKA and p75NTR promoters and affects neuroblastoma malignancy by inhibiting the cell response to NGF. Cancer Res 71:404-412.

19. Kari L, Loboda A, Nebozhyn M et al (2003) Classification and prediction of survival in patients with the leukemic phase of cutaneous $\mathrm{T}$ cell lymphoma. $\mathrm{J}$ Exp Med 197:1477-1488. 20.

20. Krejsgaard T, Odum N, Geisler C et al (2012) Regulatory T cells and immunodeficiency in mycosis fungoides and Sézary syndrome. Leukemia 26:424-432.

21. Kim EJ, Hess S, Richardson SK et al (2005) Immunopathogenesis and therapy of cutaneous T cell lymphoma. J Clin Investig 115:798-812. 22.

22. Kim S-N, Kim NH, Lee W et al (2009) Histone deacetylase inhibitor induction of P-glycoprotein transcription requires both histone deacetylase 1 dissociation and recruitment of CAAT/enhancer binding protein beta and PCAF to the promoter region. Mol Cancer Res 7:735-744. 23.

23. Lee CS, Ungewickell A, Bhaduri A et al (2012) Transcriptome sequencing in Sezary syndrome identifies Sezary cell and mycosis fungoides-associated lncRNAs and novel transcripts. Blood 120:3288-3297. 24.

24. Livak KJ, Schmittgen TD (2001) Analysis of relative gene expression data using real-time quantitative PCR and the 2(-Delta Delta C(T)) Method. Methods San Diego Calif 25:402-408.

25. Mir MA, Majee S, Das S, Dasgupta D (2003) Association of chromatin with anticancer antibiotics, mithramycin and chromomycin A3. Bioorg Med Chem 11:2791-2801

26. Nakayama A, Odajima T, Murakami H et al (2001) Characterization of two promoters that regulate alternative transcripts in the microtubule-associated protein (MAP) 1A gene. Biochim Biophys Acta 1518:260-266

27. Narducci MG, Scala E, Bresin A et al (2006) Skin homing of Sézary cells involves SDF-1-CXCR4 signaling and down-regulation of CD26/dipeptidylpeptidase IV. Blood 107:1108-1115.

28. Ritchie ME, Phipson B, Wu D et al (2015) limma powers differential expression analyses for RNA-sequencing and microarray studies. Nucleic Acids Res 43:e47.

29. Rivero S, Ruiz-García A, Díaz-Guerra MJM et al (2011) Characterization of a proximal Sp1 response element in the mouse Dlk2 gene promoter. BMC Mol Biol 12:52.

30. Shao Y, Gao Z, Marks PA, Jiang X (2004) Apoptotic and autophagic cell death induced by histone deacetylase inhibitors. Proc Natl Acad Sci USA 101:18030-18035.

31. Tajrishi MM, Shin J, Hetman M, Kumar A (2014) DNA methyltransferase 3a and mitogen-activated protein kinase signaling regulate the expression of fibroblast growth factor-inducible 14 (Fn14) during denervation-induced skeletal muscle atrophy. J Biol Chem 289:19985-19999. 32.

32. Wozniak MB, Villuendas R, Bischoff JR et al (2010) Vorinostat interferes with the signaling transduction pathway of $\mathrm{T}$-cell receptor and synergizes with phosphoinositide-3 kinase inhibitors in cutaneous T-cell lymphoma. Haematologica 95:613-621. 
33. Wu J, Nihal M, Siddiqui J et al (2009) Low FAS/CD95 expression by CTCL correlates with reduced sensitivity to apoptosis that can be restored by FAS upregulation. J Investig Dermatol 129:1165-1173. 34.

34. Zhang Q, Nowak I, Vonderheid EC et al (1996) Activation of Jak/STAT proteins involved in signal transduction pathway mediated by receptor for interleukin 2 in malignant $\mathrm{T}$ lymphocytes derived from cutaneous anaplastic large T-cell lymphoma and Sezary syndrome. Proc Natl Acad Sci USA 93:9148-9153 